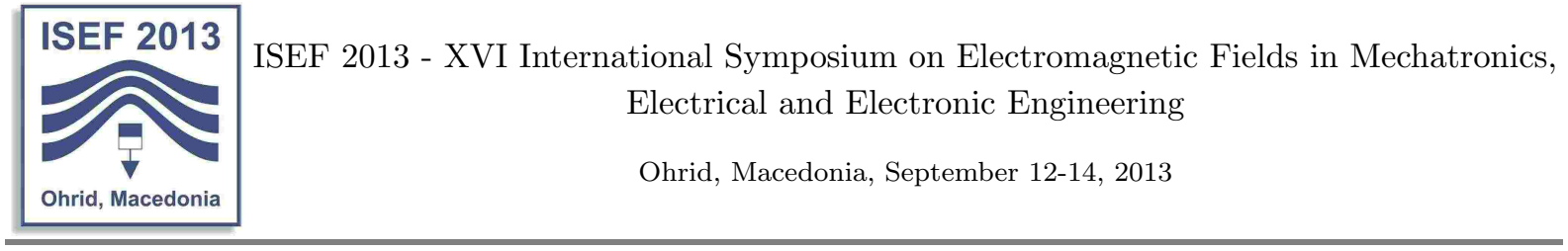

\title{
OPTIMISATION PROCESS TO SOLVE MULTIRATE SYSTEM
}

\author{
A. Pierquin, S. Brisset, T. Henneron, S. Clénet \\ EC Lille, Laboratory of Electrical Engineering and Power Electronics, \\ Cité Scientifique - CS 20048 - 59651, Villeneuve d'Ascq, France \\ email: antoine.pierquin@ec-lille.fr
}

\begin{abstract}
The modeling of a multirate system -formed by components with heterogeneous time constants- can be done using fixed-point method. This method allows a timediscretization of each subsystem with respect to its own time constant. In an optimization process, executing the loop of the fixed-point at each model evaluation can be time consuming. By adding one of the searched waveform of the system to the optimization variables, the loop can be avoided. This strategy is applied to the optimization of a transformer.
\end{abstract}

\section{Introduction}

In the context of the optimization of a multi-physic device, it is necessary to simulate several physics phenomena or several subsystems, and to couple them. An optimization process leads to a large number of evaluations of the device model. So the optimization procedure will be even longer than the device model is time consuming. For static problems, Multidisciplinary Optimization (MDO) has already been treated $[1,2,3]$. On one hand, the Multidisciplinary Feasibility (MDF) concept is an optimization process where the consistency of the modeling is ensured at each evaluation by using an iterative process between the different subsystems (fixed-point, quasi-Newton,...). On the other hand, to avoid performing the fixed-point loop and to incorporate more deeply the simulation into the optimization process, the loop is broken and the consistency of the result is guaranteed by addition of constraints into the optimization problem: this is the Individual Disciplinary Feasibility principle (IDF) [4]. In the case of dynamic systems, the same procedure can be considered: an IDF strategy where waveforms are added to the optimization variables.

\section{Multidisciplinary optimization}

Consider an optimization problem which requires to model a multirate system - a system where subsystems have different time constants. An efficient way to model such a system is the Waveform Relaxation Method (WRM) [5, 6, 7], that is a fixed-point technique applied to waveforms. This way, the model is consistent and its evaluation gives the correct behavior of the multirate system. Performing the fixed-point loop at each iteration of the optimization process could be time-consuming. This way of performing optimization is the MDF principle. The IDF principle consists in avoiding the loop by adding the waveforms to the optimization variables on one side, 
and constraints to ensure the consistency of the model on other side. The additional constraints are the relation that fixed-point has to verify.

\section{Waveform Relaxation Method}

The multirate system is composed of $r$ subsystems verifying for all $i \in\{1, \ldots, r\}$ :

$$
\begin{aligned}
& \dot{\boldsymbol{y}}_{i}(t)=\boldsymbol{f}_{i}(\boldsymbol{y}(t), \boldsymbol{z}(t)), \\
& \boldsymbol{g}(\boldsymbol{y}(t), \boldsymbol{z}(t))=\mathbf{0} .
\end{aligned}
$$

With

$$
\begin{gathered}
t \in T=\left[t_{0}, t_{f}\right], \quad \boldsymbol{y}_{i}: T \rightarrow \mathbf{R}^{m_{i}}, \quad \boldsymbol{z}_{i}: T \rightarrow \mathbf{R}^{n_{i}}, \\
\sum_{i=1}^{r} m_{i}=m, \quad \sum_{i=1}^{r} n_{i}=n, \\
\boldsymbol{y}=\left[\boldsymbol{y}_{1}, \ldots, \boldsymbol{y}_{r}\right]^{t}, \quad \boldsymbol{z}=\left[\boldsymbol{z}_{1}, \ldots, \boldsymbol{z}_{r}\right]^{t}, \\
\boldsymbol{f}_{i}:\left(\mathbf{R}^{n}, \mathbf{R}^{m}\right) \rightarrow \mathbf{R}^{m_{i}}, \quad \boldsymbol{g}_{i}:\left(\mathbf{R}^{n}, \mathbf{R}^{m}\right) \rightarrow \mathbf{R}^{n_{i}} .
\end{gathered}
$$

The principle of the WRM is to perform a relaxation between the waveforms of the different subsystems. It produces iteratively an approximation $\left(\boldsymbol{y}^{k}, \boldsymbol{z}^{k}\right)$ of the exact solution $(\boldsymbol{y}, \boldsymbol{z})$. Several relaxation schemes exist, we present here the Gauss-Seidel scheme. At each iteration $k$, the following subsystems are solved sequentially:

$$
\begin{aligned}
& \dot{\boldsymbol{y}}_{i}^{k}(t)=\boldsymbol{f}_{i}\left(\tilde{\boldsymbol{y}}_{i}^{k}(t), \tilde{\boldsymbol{z}}_{i}^{k}(t)\right), \\
& \boldsymbol{g}\left(\tilde{\boldsymbol{y}}_{i}^{k}(t), \tilde{\boldsymbol{z}}_{i}^{k}(t)\right)=\mathbf{0},
\end{aligned}
$$

where $\tilde{\boldsymbol{y}}_{i}^{k}=\left[\boldsymbol{y}_{1}^{k}, \ldots, \boldsymbol{y}_{i-1}^{k}, \boldsymbol{y}_{i}^{k}, \boldsymbol{y}_{i+1}^{k-1}, \ldots, \boldsymbol{y}_{r}^{k-1}\right]$ and $\tilde{\boldsymbol{z}}_{i}^{k}=\left[\boldsymbol{z}_{1}^{k}, \ldots, \boldsymbol{z}_{i-1}^{k}, \boldsymbol{z}_{i}^{k}, \boldsymbol{z}_{i+1}^{k-1}, \ldots, \boldsymbol{z}_{r}^{k-1}\right]$.

The iterative process stops when the difference between two iterates is small enough, i.e. when $\left\|\begin{array}{l}\boldsymbol{y}^{k}-\boldsymbol{y}^{k-1} \\ \boldsymbol{z}^{k}-\boldsymbol{z}^{k-1}\end{array}\right\|$ is less than a given tolerance. So the WRM appears to be a fixed-point process: indeed, if we denote by $\boldsymbol{\Phi}$ the operator that gives the iterate $k$ from the previous iterate $k-1$, we have that: $\left(\boldsymbol{y}^{k}, \boldsymbol{z}^{k}\right)=\boldsymbol{\Phi}\left(\boldsymbol{y}^{k-1}, \boldsymbol{z}^{k-1}\right)$. Now, we want to have $\left(\boldsymbol{y}^{k}, \boldsymbol{z}^{k}\right)=\left(\boldsymbol{y}^{k-1}, \boldsymbol{z}^{k-1}\right)$, equivalent to $\left(\boldsymbol{y}^{k-1}, \boldsymbol{z}^{k-1}\right)=\boldsymbol{\Phi}\left(\boldsymbol{y}^{k-1}, \boldsymbol{z}^{k-1}\right)$. So WRM solves the fixed-point problem:

$$
\Phi(\chi)=\chi, \quad \chi=(\boldsymbol{y}, \boldsymbol{z}) .
$$

Let us consider that a model simulated by WRM is used for an optimization; then, at each evaluation of the model, the subsystems from $i=1$ to $r$ are solved at each iteration $k$ until that convergence is effective. If we denote by $K$ the minimum number of iterations required to obtain convergence, each subsystem has to be solved at least $K$ times per model evaluation.

\section{$\underline{\text { Individual Disciplinary Feasibility }}$}

At each evaluation of a WRM modeling, subsystems are solved several times on the time-domain $T=\left[t_{0}, t_{f}\right]$ during the loop. At the end of the loop, the fixed-point criterion $\boldsymbol{\Phi}(\boldsymbol{\chi})=\boldsymbol{\chi}$ is satisfied. The idea of the IDF is to avoid performing this loop during the optimization process. At each evaluation of the model, subsystems are solved only one times; consistency of the final 
result is assured by the optimization process by adding the fixed-point condition $\mathbf{\Phi}(\chi)=\chi$ to the constraints.

Let us consider the optimization problem:

$$
\tilde{\boldsymbol{x}}=\arg \min _{\boldsymbol{x}} f(\boldsymbol{x}) \quad \text { such that } \quad \boldsymbol{k}_{f}(\boldsymbol{x}) \leq \mathbf{0} .
$$

Suppose that evaluation of $f$ or $\boldsymbol{k}_{f}$ requires to evaluate a multirate system. The modeling of this system can be done by using WRM. But we saw that it will imply to solve $K$ times each subsystem per evaluation of the model. IDF suggests to add variables and constraints to the optimization problem, to not perform the loop: the waveforms $\chi$ are now a part of the optimization variables, and the relation $\boldsymbol{\Phi}(\chi)=\chi$ a part of the constraints. The IDF problem is expressed as follow:

$$
(\tilde{\boldsymbol{x}}, \tilde{\boldsymbol{\chi}})=\arg \min _{(\boldsymbol{x}, \boldsymbol{\chi})} f(\boldsymbol{x}, \boldsymbol{\chi}) \quad \text { such that } \quad\left\{\begin{array}{l}
\boldsymbol{k}_{f}(\boldsymbol{x}, \boldsymbol{\chi}) \leq \mathbf{0}, \\
\mathbf{\Phi}(\boldsymbol{\chi})-\chi=\mathbf{0} .
\end{array}\right.
$$

By this way, $\boldsymbol{\Phi}$ is evaluated only once per model evaluation (Fig. 1(b)), whereas WRM implies to evaluate $\boldsymbol{\Phi}$ until convergence of the fixed-point iteration (Fig. 1(a)). However, the number of optimization variables is strongly increased and we have to deal with a curse of dimensionality.

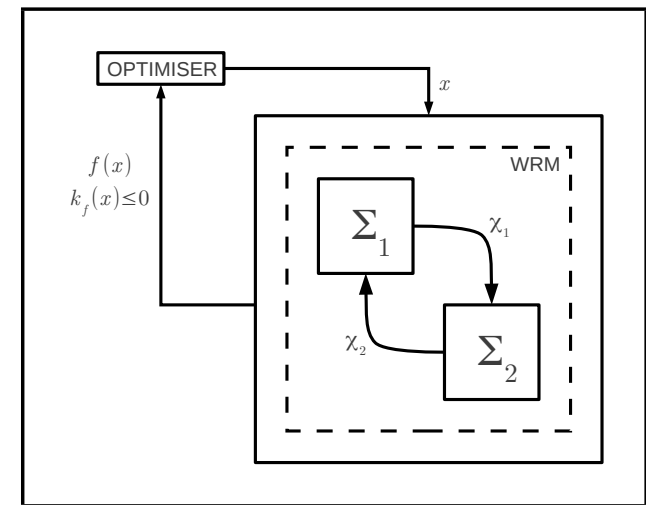

(a) Optimization with WRM modeling

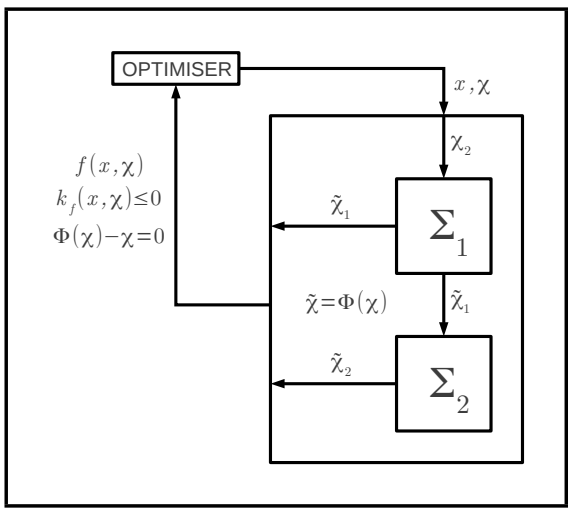

(b) Optimization with IDF

Fig. 1: Optimization with WRM modeling or IDF principle.

\section{Application}

We study a device formed of an LC filter supplying a transformer (Fig. 2(a)). This device can be split into two parts with time-constants very differents: the filter forms a first subsystem and the transformer a second. The filter is a voltage source, an inductor, a capacitor and a current source; the second subsystem is composed of a voltage source, a resistor and a transformer. The current source of the first subsystem is the current $i_{R}$ in the primary coil of the transformer, and the voltage source of the transformer is the voltage $v_{c}$ of the capacitor (Fig. 2(b)). Time step in the first subsystem is $d t_{1}=5 \cdot 10^{-7} \mathrm{~s}$, and $d t_{2}=1 \cdot 10^{-3} \mathrm{~s}$ in the second. In the MDF case, at each iteration $k$ of the fixed-point loop, $i_{R}^{k-1}$ is a source for the first subsystem, that is solved and gives $v_{c}^{k}$. From $v_{c}^{k}$ as voltage source, the transformer subsystem is solved to obtain $i_{R}^{k}=\Phi\left(i_{R}^{k-1}\right)$, and the loop continues until convergence, i.e. consistency. In the IDF case and for a sequential resolution of the subsystems, it is not necessary to give the voltage and the current as input waveforms, but only the current. So $i_{R}$ will be a part of the optimization variables, and the relation $\Phi\left(i_{R}\right)=i_{R}$ will be added as a constraint. 
The transformer is modeled with a 2D Finite Element Method (FEM): we use the A-formulation to express the magnetic induction. The expression

$$
B=\operatorname{curl} A
$$

is introduced into the equations:

$$
\begin{aligned}
\operatorname{curl} \boldsymbol{H} & =\boldsymbol{J} \\
\operatorname{div} \boldsymbol{B} & =0 .
\end{aligned}
$$

Voltage is imposed by the coupling equation [8]:

$$
\frac{d}{d t}\left(\int_{D} \boldsymbol{A} \cdot \boldsymbol{N} d v\right)+R \cdot i_{R}=v_{c} .
$$

After a space discretization of the magnetic vector potential $\boldsymbol{A}$ using edge shape functions [9], we obtain the following matrix system to solve:

$$
\boldsymbol{M} \dot{\boldsymbol{X}}+\boldsymbol{P} \boldsymbol{X}=\boldsymbol{V} .
$$

The system is then solved by using an implicit Euler time-scheme discretization.

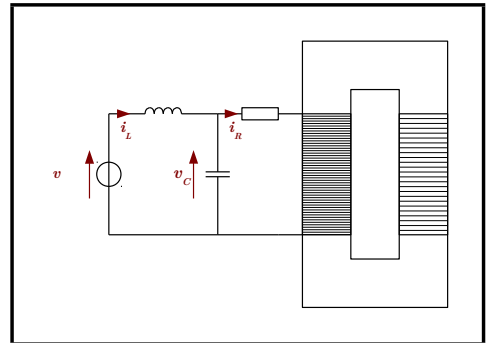

(a) Complete device

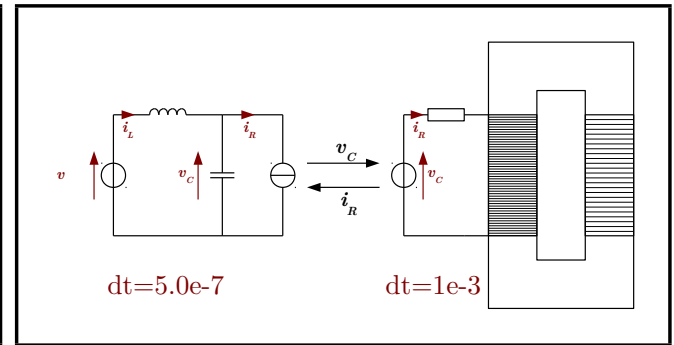

(b) Split device

Fig. 2: Complete and split device.

We want to minimize the transformer mass $m$ and fix the root mean square (RMS) current $i_{r m s}$ in the transformer's coil. The transformer geometry is entirely defined by its height $H$ and its width $L$ (Fig. 3 ).

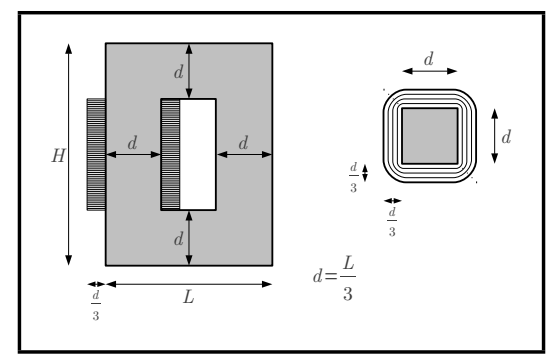

Fig. 3: Transformer's geometry.

The problem to solve is the following:

$$
\left\{\begin{array}{l}
\min _{H, L} m(H, L) \\
20 \mathrm{~cm} \leq H \leq 40 \mathrm{~cm} \\
12 \mathrm{~cm} \leq L \leq 24 \mathrm{~cm} \\
H-\frac{2 L}{3}>0 \\
i_{r m s}=3 A
\end{array}\right.
$$


In this case, $i_{r m s}$ is obtained by modeling the device on a time-domain $T=\left[t_{0}, t_{f}\right]$ by WRM. With an IDF strategy, the problem to solve becomes:

$$
\left\{\begin{array}{l}
\min _{H, L, i_{R}} m\left(H, L, i_{R}\right), \\
20 \mathrm{~cm} \leq H \leq 40 \mathrm{~cm} \\
12 \mathrm{~cm} \leq L \leq 24 \mathrm{~cm} \\
H-\frac{2 L}{3}>0 \\
i_{r m s}=3 A \\
\Phi\left(i_{R}\right)=i_{R}
\end{array}\right.
$$

The size of the optimization variables is raised, but the optimization process will deal with the consistency constraint, there is no fixed-point loop anymore. The current $i_{R}$ is discretized in time with 100 points, so IDF problem has 102 optimization variables: $H, L, i_{R}$. Figures 4 present the results of the optimization for the problems MDF (13) and IDF (14) from the same initial point for the geometry variables. We use a Sequential Quadratic Programming (SQP) for the optimizations but we consider that gradient of the function is available, and so there is no estimation of the gradient by finite differences. The IDF optimization converges for all points to a solution very close to the solution of MDF problem, as shown in Figure 4(a) for the optimal variables and in Figure 4(b) for the optimal mass. Figure 4(c) shows that the number of iterations done by SQP algorithm is quite similar for both formulation, but the number of evaluations of the $\Phi$-operator is more important in the case of MDF formulation (Figure 4(d)). Indeed, only one evaluation is performed by iteration for IDF optimization, whereas at least 6 evaluations are necessary for MDF optimization. So if the gradient is available at a computing time similar to the model's one, IDF could allow to perform an optimization in a shorter time than the classic MDF formulation.

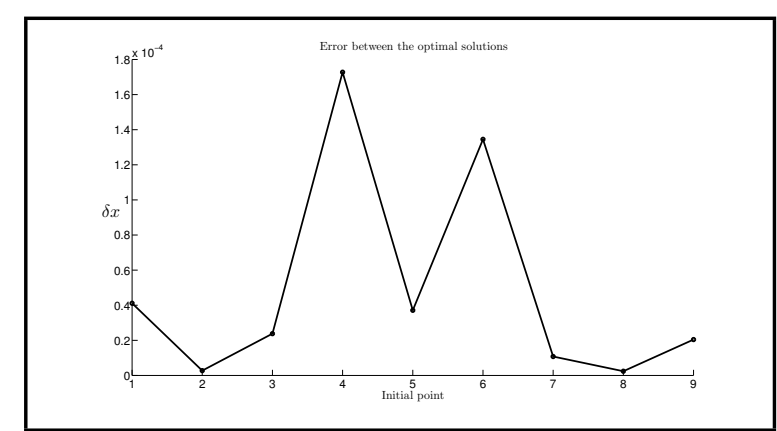

(a) Error between optimal solutions: $\frac{\left\|x_{I D F}-x_{M D F}\right\|}{\left\|x_{M D F}\right\|}$.

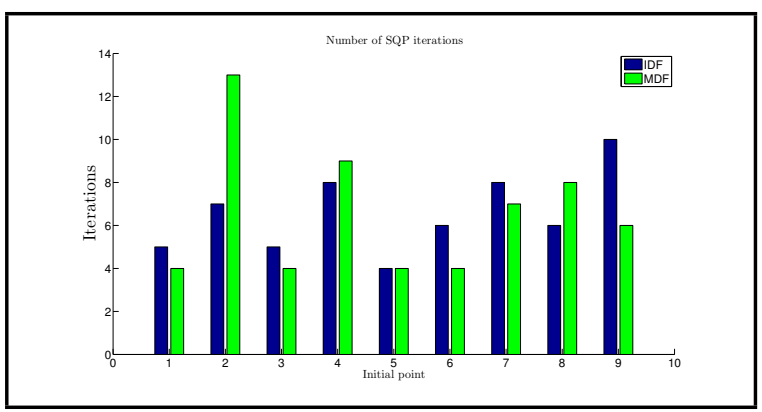

(c) Number of SQP iterations.

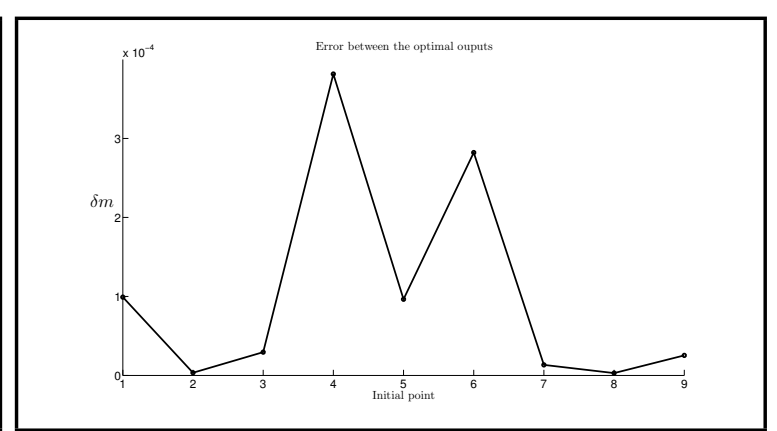

(b) Error between optimal outputs: $\frac{\left\|m_{I D F}-m_{M D F}\right\|}{\left\|m_{M D F}\right\|}$.

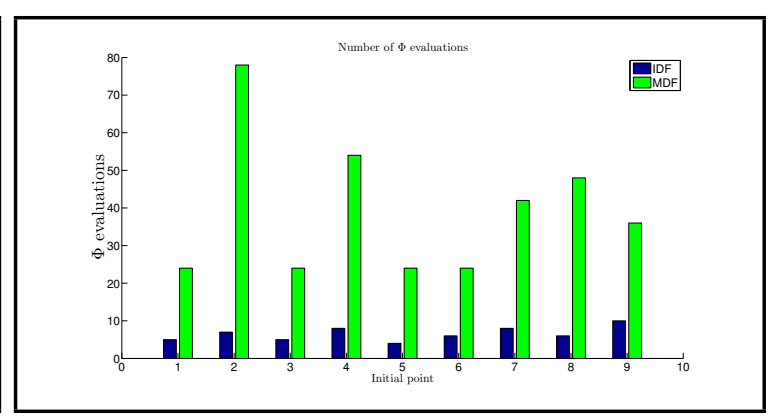

(d) Number of evaluations of the $\Phi$-operator.

Fig. 4: Comparison of results from IDF optimization and MDF optimization. 


\section{Conclusion}

The Waveform Relaxation Method is a fixed-point process that allows to simulate multirate system. But the fixed-point loop to ensure the model consistency can be time consuming. If the model is used in an optimization process, this loop can be avoid by adding some optimization variables and by considering the fixed-point criterion as a constraint of the optimization problem: this is the Individual Disciplinary Feasibility optimization. If the computation of gradients could be avoid, IDF could accelerate the optimization process as shown on the optimization of a transformer supplied by an LC filter.

\section{$\underline{\text { References }}$}

[1] J. Sobieszczanski-Sobieski and R.T. Haftka. Multidisciplinary aerospace design optimization: survey of recent developments. Structural optimization, 14:1-23, 1997.

[2] R. D. Braun and I. M. Kroo. Development and application of the collaborative optimization architecture in a multidisciplinary design environment. In Multidisciplinary Design Optimization: State of the Art, pages 98-116. SIAM, 1997.

[3] R. Ben-Ayed and S. Brisset. Improvement of output space mapping by using multidisciplinary optimization formulations. COMPEL, march 2011.

[4] S. Kreuawan, F. Gillon, and P. Brochet. Optimal design of permanent magnet motor using multidisciplinary design optimization. In Electrical Machines, 2008. ICEM 2008. 18th International Conference on, pages 1-6, 2008.

[5] M.L. Crow and M.D. Ilic. The waveform relaxation method for systems of differential/algebraic equations. In Decision and Control, 1990., Proceedings of the 29th IEEE Conference on, pages 453 -458 vol.2, dec 1990.

[6] E. Lelarasmee, A.E. Ruehli, and A.L. Sangiovanni-Vincentelli. The waveform relaxation method for time-domain analysis of large scale integrated circuits. Computer-Aided Design of Integrated Circuits and Systems, IEEE Transactions on, 1(3):131 - 145, july 1982.

[7] S. Schops, H. De Gersem, and A. Bartel. A cosimulation framework for multirate time integration of field/circuit coupled problems. Magnetics, IEEE Transactions on, 46(8):3233 -3236, aug. 2010.

[8] F. Piriou and A. Razek. Finite element analysis in electromagnetic systems-accounting for electric circuits. Magnetics, IEEE Transactions on, 29(2):1669-1675, 1993.

[9] Alain Bossavit. A rationale for 'edge-elements' in 3-d fields computations. Magnetics, IEEE Transactions on, 24(1):74-79, 1988. 